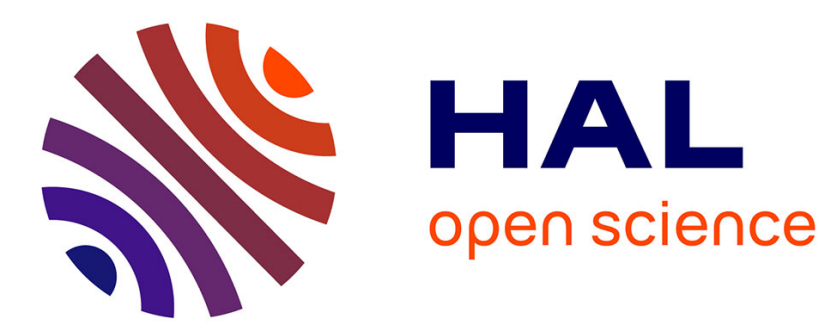

\title{
New models for the location of controversial facilities: A bilevel programming approach
}

\author{
Martine Labbé, Marina Leal, Justo Puerto
}

\section{To cite this version:}

Martine Labbé, Marina Leal, Justo Puerto. New models for the location of controversial facilities: A bilevel programming approach. Computers and Operations Research, 2019, 107, pp.95 -106. 10.1016/j.cor.2019.03.003 . hal-01933601

\section{HAL Id: hal-01933601 \\ https://hal.inria.fr/hal-01933601}

Submitted on 23 Nov 2018

HAL is a multi-disciplinary open access archive for the deposit and dissemination of scientific research documents, whether they are published or not. The documents may come from teaching and research institutions in France or abroad, or from public or private research centers.
L'archive ouverte pluridisciplinaire HAL, est destinée au dépôt et à la diffusion de documents scientifiques de niveau recherche, publiés ou non, émanant des établissements d'enseignement et de recherche français ou étrangers, des laboratoires publics ou privés. 


\title{
New models for the location of controversial facilities: A bilevel programming approach
}

\author{
Martine Labbé ${ }^{\dagger}$, Marina Leal ${ }^{\ddagger}$ and Justo Puerto ${ }^{\ddagger}$ \\ †Université Libre de Bruxelles \\ ${ }_{\ddagger}^{\ddagger}$ IMUS, Universidad de Sevilla
}

\begin{abstract}
Motivated by recent real-life applications in Location Theory in which the location decisions generate controversy, we propose a novel bilevel location model in which, on the one hand, there is a leader that chooses among a number of fixed potential locations which ones to establish. Next, on the second hand, there is one or several followers that, once the leader location facilities have been set, chooses his location points in a continuous framework. The leader's goal is to maximize some proxy to the weighted distance to the follower's location points, while the follower(s) aim is to locate his location points as close as possible to the leader ones. We develop the bilevel location model for one follower and for any polyhedral distance, and we extend it for several followers and any $\ell_{p}$-norm, $p \in \mathbb{Q}, p \geq 1$. We prove the NP-hardness of the problem and propose different mixed integer linear programming formulations. Moreover, we develop alternative Benders decomposition algorithms for the problem. Finally, we report some computational results comparing the formulations and the Benders decompositions on a set of instances.
\end{abstract}

Keywords: Bilevel optimization, locational analysis, combinatorial optimization.

\section{InTRODUCTION}

Location is a research area devoted to the optimal placement of facilities $[2,6$, $12,13,14,19,21]$, including among many others emergency systems $[4,6,24]$, service providers [1,5], infrastructures, etc., and it is a basic building block of most transportation, communication or logistic problems. An optimal location can be 
chosen according to different criteria depending on the rationale behind the considered model. The most popular ones are the minimization of the total or maximum transportation cost $[2,6]$, the maximization of some coverage goal $[1,4,5,6]$, or the minimization of the undesirable effects induced by the facilities $[7,8,12]$.

Location Theory includes a number of real-life applications in which the location decisions generate controversy. This controversy must be understood as a disagreement among users with different, non-aligned or opposite interests. Examples of this controversial location can be found in the literature, for example, in the areas of semiobnoxious facility location or in problems that involve the location and protection of critical infrastructures or facilities sensitive to intentional attacks.

In the last decades, the consciousness-raising in environmental issues has grown substantially, specially in those aspects that affect human health or have adverse effects on people. As a consequence of this awareness-raising, the location of (semi-) obnoxious facilities has been extensively studied. Obnoxious facilities are those that generate a disservice to the people nearby while producing an intended product or service [10]. However, if only these undesirable effects are taken into account when locating them, these facilities would never be opened or would be located too far from the population centers making use of the produced services, thus generating huge costs. For that reason, in the last years, there has been an increasing focus in analyzing the problem of locating semi-obnoxious facilities [7, 12, 18]. Semiobnoxious facilities has been defined as useful but unwelcome facilities that produce environmental concerns. That is, facilities that population centers (demand points) want them away, but there are some interests (political, economical ...) in locating them close the demand points, generating in this way, location controversy. Classical examples of this kind of facilities are chemical and power plants, airports, waste dumps, detoxification centers, etc., as listed in [18].

Another area that has also attracted increasing attention of researchers in the last years is the location and protection of vulnerable facilities (with high risk of disruption) and the protection of critical facilities, including not only those related to disruptions produced by natural disasters or natural failures, but also those referred to disruptions produced by man-made attacks $[8,9,23]$. Critical infrastructure is a term to describe assets that are essential for the functioning of a society and economy. 
Most commonly associated with the term are facilities for heating, water supply, public health, security services, telecommunication, economic sector, etcetera. Clearly, the location and protection of these types of facilities generates also controversy, since the population is aware of the risks that may appear close to those facilities due to the confrontation between two antagonist parties: attackers and defenders with visibly opposite goals.

The above-mentioned problems have been usually addressed via biobjective (multiobjective) approaches, difference of objective functions, maximin optimization and, if there exists a hierarchical structure in the decision-making process, by means of bilevel optimization.

Motivated by the increasing interest in real-world applications generating location controversy, we introduce a new model for its study and analysis.

The situation that we want to address models the existence of two parties acting sequentially in a decision-making process. On the one hand, there is a leader who wants to locate some primary facilities and must choose among a number of fixed potential locations where to establish them. On the other hand, there is one (or several) follower(s) that, once the primary facilities have been set, chooses the placement of some secondary facilities, in a continuous environment. The leader's goal is to maximize some proxy of the overall weighted distance to the follower's secondary facility locations. Meanwhile, the follower(s) aim is to locate their secondary facilities as close as possible to the primary ones, minimizing a cost proportional to the distance from the secondary facilities to the primary ones set by the leader.

The reader may observe that this model fits perfectly to the cases mentioned above.

The paper is structured as follows. The following section introduces the model, sets the notation and proves the NP-hardness of the considered problem. In Section 3 , we develop the mathematical programming formulations and resolution algorithms for the problem with one follower and any block norm. Two different approaches, based on the representation of the norms, have been considered. Furthermore, due to their importance, they have been applied to the case of the $\ell_{1}$ and $\ell_{\infty}$ norm. In the next Section 4, we extend the model to several followers and non-polyhedral norms. Section 5 is devoted to the computational study of the different methods discussed in the previous sections. Finally, Section 6 concludes the paper. 
2. THE MODEL

We consider a situation with two different types of location entities: the primary facilities (critical infrastructures, goods to protect, demand-points, etc.), and the secondary facilities (terrorists nets, thefts, semi-obnoxious facilities, detoxification centers, recycling or power plants, etc.). The primary facilities wish to be located as far as possible from the secondary facilities, meanwhile the secondary facilities aim to be located as close as possible to the primary ones. The model we present consists in choosing the location of the primary facilities (these are set first), taking into account that, the secondary facilities will be located afterwards knowing their location. For the ease of presentation, we restrict ourselves to the case where a unique secondary facility will be located. The reader is referred to Section 4 for the extension to several facilities.

We will model this hierarchical structure using Bilevel Optimization. Bilevel programming targets hierarchical optimization problems in which part of the constraints translate the fact that some of the variables constitute an optimal solution of another optimization problem. There exist a leader that acts first, and then a follower that reacts to the leader's decision.

We assume that there is a leader (setting the primary facilities) that chooses among a set of potential locations $B$ the placement of some new primary facilities. We also consider that there is a set $N B$ of primary facilities already established, and there exists a budget constraint on the overall investment for the location of the new primary facilities. On the other hand, once the primary facilities are set, the follower chooses the location of the secondary facility in a continuous framework. The proximity between the primary and secondary facilities is measured as a weighted sum of a distance to all primary facilities.

We denote by $c_{j}$ the cost of opening the primary facility $j$, for all $j \in B$, by $C$ the maximum budget, by $f_{j} \in \mathbb{R}^{n}$ the given location point $j \in B \cup N B$, and by $w_{j}$ the weight factor that scales the distance from the secondary facility to $f_{j}$ according to its importance. We define the binary decision variables $y_{j}=1$ if $f_{j}, j \in B$, is open, and $y_{j}=0$ otherwise.

For the follower problem we define the decision variable $x \in \mathbb{R}^{n}$ that specifies the location of the secondary facility. 
Therefore, the bilevel problem can be modeled as:

(BLP)

$$
\begin{aligned}
\max & \sum_{j \in B} w_{j} d\left(x, f_{j}\right) y_{j}+\sum_{j \in N B} w_{j} d\left(x, f_{j}\right) \\
\text { st. } & \sum_{j \in B} c_{j} y_{j} \leq C, \\
& y_{j} \in\{0,1\} \quad j \in B, \\
& x \in \arg \min _{x} \sum_{j \in B} w_{j} d\left(x, f_{j}\right) y_{j}+\sum_{j \in N B} w_{j} d\left(x, f_{j}\right),
\end{aligned}
$$

where $d\left(x, f_{j}\right)$ denotes any distance induced by some norm:

$$
d\left(x, f_{j}\right)=\left\|x-f_{j}\right\| .
$$

Observe that BLP is a bilevel max-min problem, in which the objective function of both levels is a proxy of the distance between the primary and the secondary facilities. The resulting bilevel problem contains a knapsack problem at the upper level, and a continuous single-facility location problem at the lower level.

To state the complexity of the problem, we provide the following result.

Theorem 1. The bilevel location model $\boldsymbol{B L P}$ is NP-hard.

Proof. Let us consider the distance induced by a norm $\|\cdot\|$, and an instance such that $|N B|=1$ and $w_{j^{0}}>\sum_{j \in B} w_{j}$ for $j^{0} \in N B$.

For this instance, we know, using the majority theorem, see for example [15], that the optimal solution of the continuous location problem is $x^{*}=f_{j}^{0}$. Then, if we denote $r_{j}^{*}=\left\|f_{j}^{0}-f_{j}\right\|$, Problem BLP can be written as:

$$
\begin{aligned}
& \max \sum_{j \in B} w_{j} y_{j} r_{j}^{*} \\
& \text { s.t. } \\
& \sum_{j \in B} c_{j} y_{j} \leq C, \\
& \quad y_{j} \in\{0,1\} \forall j \in B,
\end{aligned}
$$

which is a knapsack problem, known to be NP-hard [11]. 


\section{Mathematical programming Formulations AND RESOLUtion}

\section{ALGORITHMS}

This section is devoted to present useful mathematical programming formulations for BLP in order to solve it with off-the-self solvers. In addition, we will present alternative add-hoc algorithms, based on decompositions, that prove to be more efficient than the solvers acting on the Mixed Integer Linear Programming (MILP) above-mentioned formulations.

For the sake of presentation, we assume in this section that we measure distances via block norms. The family of block norms, also called polyhedral norms, includes all norms $\|.\|_{P}$ whose unit ball $P$ is a symmetric with respect to the origin, convex bounded polyhedral set containing the origin in its interior. We will denote then by $\operatorname{ext}(P)$ the set of extreme points of $P$ and by $P^{o}$ : the polar set of $P$, that is, $P^{o}:=\left\{x \in \mathbb{R}^{n}:\langle x, p\rangle \leq 1, \forall p \in P\right\}$. The reader may note that the commonly used $\ell_{1}$ and $\ell_{\infty}$ norms belong to this family.

In order to deal with the problem we develop two different procedures: the first one is based on the evaluation of the norm through its primal expression, using its unit ball defined by $P$, and the second one evaluates the norm through its dual expression, using its dual unit ball $P^{o}$.

These two different forms used to handle the problem are justified, as we will see, by the fact that depending on the cases one can be more efficient than the other due to the structure of the set of extreme points of $P$ and $P^{o}$. We will illustrate this behavior in the following sections with the $\ell_{1}$ and $\ell_{\infty}$ norms.

3.1. First approach: Evaluating norms with its primal expression. It is well-known (see for example [20] and [22]), that the value of $\|x\|_{P}$ is given as:

\section{(PrimalNormP)}

$$
\begin{aligned}
\|x\|_{P}=\min & \sum_{b \in \operatorname{ext}(P)} \mu_{b}, \\
\text { s.t. } & x=\sum_{b \in \operatorname{ext}(P)} \mu_{b} b, \\
& \mu_{b} \geq 0, \quad b \in \operatorname{ext}(P) .
\end{aligned}
$$


This representation of the norm gives rise to a trilevel problem. Using such representation we develop a MILP Formulation and a Benders like algorithm in order to solve BLP, using off-the-shelf solvers.

3.1.1. A MILP formulation. Let us assume that $x=\left(x_{1}, \ldots, x_{n}\right), f_{j}=\left(f_{j 1}, \ldots, f_{j n}\right)$, for all $j \in B \cup N B$ and $b=\left(b_{1}, \ldots, b_{n}\right)$ for all $b \in \operatorname{ext}(P)$. By representing $\|x\|_{P}$ as in PrimalNormP, BLP can be written as the following bilevel problem:

(BLP-P)

$$
\begin{aligned}
\max & \sum_{j \in B} w_{j} y_{j} r_{j}+\sum_{j \in N B} w_{j} r_{j} \\
\text { s.t. } & \sum_{j \in B} c_{j} y_{j} \leq C, \\
& y_{j} \in\{0,1\} \quad j \in B, \\
& r \in \arg \min _{x, r} \sum_{j \in B} w_{j} y_{j} r_{j}+\sum_{j \in N B} w_{j} r_{j}, \\
& r_{j}=\sum_{b \in \operatorname{ext}(P)} \mu_{b}^{j}, \quad j \in B \cup N B, \\
& x_{i}=\sum_{b \in e x t(P)} \mu_{b}^{j} b_{i}+f_{j i}, \quad j \in B \cup N B, i=1, \ldots, n, \\
& \mu_{b}^{j} \geq 0, \quad b \in \operatorname{ext}(P), j \in B \cup N B, \\
& r_{j} \geq 0, \quad j \in B \cup N B, \\
& x_{i} \in \mathbb{R}, \quad i=1, \ldots, n .
\end{aligned}
$$

In the above formulation, variables $r_{j}$, defined in constraint (4), represent the distance between $x$ and $f_{j}$ and constraints (5) set the correct representation of coordinates of the secondary facility in terms of the reference system induced by $f_{j}$ and the extreme points of $P$. Constraints (1) and (2), as in BLP, are knapsack constraints, corresponding to the choice of the location of the primary facilities, according to a budget constraint. Constraints (3)-(8) define the lower level problem, the continuous location problem, in which the representation of the norm has been included. 
Proposition 1. Problem $\boldsymbol{B L P}-\boldsymbol{P}$ can be reformulated as the following single level problem $\boldsymbol{B L} \boldsymbol{P}-\boldsymbol{P}$ '.

(BLP-P')

$$
\max \sum_{j \in B} w_{j} y_{j} r_{j}+\sum_{j \in N B} w_{j} r_{j}
$$

$$
\begin{aligned}
& \text { s.t. } \sum_{j \in B} c_{j} y_{j} \leq C, \\
& y_{j} \in\{0,1\} \quad j \in B, \\
& \sum_{j \in B} w_{j} y_{j} r_{j}+\sum_{j \in N B} w_{j} r_{j}=\sum_{i=1}^{n} \sum_{j \in B \cup N B} \beta_{j i} f_{j i} \\
& r_{j}=\sum_{b \in e x t(P)} \mu_{b}^{j}, \quad j \in B \cup N B, \\
& x_{i}=\sum_{b \in e x t(P)} \mu_{b}^{j} b_{i}+f_{j i}, \quad j \in B \cup N B, i=1, \ldots, n, \\
& \mu_{b}^{j} \geq 0, \quad b \in e x t(P), j \in B \cup N B, \\
& r_{j} \geq 0, \quad j \in B \cup N B, \\
& x_{i} \in \mathbb{R}, \quad i=1, \ldots, n, \\
& \alpha_{j} \leq w_{j} y_{j}, \quad j \in B, \\
& \alpha_{j} \leq w_{j}, \quad j \in N B, \\
& \quad \sum_{j \in B \cup N B} \beta_{j i}=0, \quad i=1, \ldots, n, \\
&-\alpha_{j}-\sum_{i=1}^{n} b_{i} \beta_{j i} \leq 0, \quad j \in B \cup N B, b \in \operatorname{ext}(P) .
\end{aligned}
$$

Proof. Given a solution $y$, representing a feasible set of locations for the primary facilities in BLP-P, the inner problem in BLP-P is a feasible Linear Program (LP) with finite solution, and its dual is:

(Dual-P)

$$
\begin{aligned}
& \max \sum_{i=1}^{n} \sum_{j \in B \cup N B} \beta_{j i} f_{j i} \\
& \text { s.t. } \alpha_{j} \leq w_{j} y_{j}, \quad j \in B,
\end{aligned}
$$




$$
\begin{aligned}
& \alpha_{j} \leq w_{j}, \quad j \in N B, \\
& \sum_{j \in B \cup N B} \beta_{j i}=0, \quad i=1, \ldots, n, \\
& -\alpha_{j}-\sum_{i=1}^{n} b_{i} \beta_{j i} \leq 0, \quad j \in B \cup N B, b \in \operatorname{ext}(P) .
\end{aligned}
$$

Then, Problem BLP-P is equivalent to the single level formulation BLP-P' since constraint (9) is the strong duality condition stating that the primal and dual objectives of the lower level problem must be equal, and the blocks of constraints (1)-(2), (4)-(8) and (10)-(13) represent, respectively, the upper level problem constraints, the lower level primal problem constraints and the lower level dual problem constraints.

We can observe that the above formulation contains some bilinear terms: $r_{j} y_{j}$. In order to transform that formulation into a mixed integer linear problem, the bilinear terms can be linearized (see [17]) giving rise to an exact MILP formulation for the bilevel problem. To this end, we substitute the terms $r_{j} y_{j}$ by the variables $\hat{r}_{j} ; \forall j \in B \cup N B$ and add the following set of constraints:

$$
\begin{array}{ll}
\hat{r}_{j} \leq r_{j}, & j \in B \cup N B, \\
\hat{r}_{j} \leq M_{j} y_{j}, & j \in B \cup N B \\
\hat{r}_{j} \geq r_{j}-M_{j}\left(1-y_{j}\right), & j \in B \cup N B \\
\hat{r}_{j} \geq 0, & j \in B \cup N B .
\end{array}
$$

The previous block of constraints requires to set a valid value for the "big- $M$ "constants. It is easy to observe that $M_{j}$ can be chosen equal to the maximum distance between $f_{j}$ and any other point in $B \cup N B$.

3.1.2. Benders like algorithm for solving $\boldsymbol{B L} \boldsymbol{P}$. Now, we propose an alternative method to solve the bilevel location problem under a block norm which is based on a decomposition of the problem.

For a given solution $y$, the inner problem in BLP-P is an LP whose set of constraints does not depend on the variables associated to the master (leader) problem 
(does not depend on $y$ ). Then, if we denote by $\mathbb{P}$ the set of extreme points of the inner problem, solving such problem is equivalent to evaluate the objective function at the points in $\mathbb{P}$ and to take the minimum objective function value. Then, the continuous location inner problem can be rewritten as the following optimization problem:

$\max q$

$$
\text { s.t. } q \leq \sum_{j \in B} \sum_{i=1}^{n} w_{j} y_{j} r_{j}^{\tau}+\sum_{j \in N B} \sum_{i=1}^{n} w_{j} r_{j}^{\tau}, \quad \forall r^{\tau} \in \mathbb{P} .
$$

In order to apply Benders decomposition, and using the above formulation, Problem BLP-P can be reformulated as:

$\max q$

$$
\begin{array}{ll}
\text { s.t. } & \sum_{j \in B} c_{j} y_{j} \leq C, \\
& y_{j} \in\{0,1\}, \quad j \in B, \\
& q \leq \sum_{j \in B} \sum_{i=1}^{n} w_{j} y_{j} r_{j}^{\tau}+\sum_{j \in N B} \sum_{i=1}^{n} w_{j} r_{j}^{\tau}, \quad \forall r^{\tau} \in \mathbb{P} .
\end{array}
$$

Our approach to solve the above problem is to sequentially identify and add extreme points in $\mathbb{P}$ to the problem until a certificate of optimality is fulfilled (eventually in the worse case after adding all extreme points).

To describe the algorithm, we denote by $\mathcal{P}$ a subset of points in $\mathbb{P}$. With the purpose of obtaining upper bounds for BLP-P, in the algorithm, we define the following Master Problem:

$(\mathrm{MP})$

$\max q$

$$
\text { s.t. } q \leq \sum_{j \in B} \sum_{i=1}^{n} w_{j} y_{j} r_{j}^{\tau}+\sum_{j \in N B} \sum_{i=1}^{n} w_{j} r_{j}^{\tau}, \quad r^{\tau} \in \mathcal{P},
$$




$$
\begin{aligned}
& \sum_{j \in B} c_{j} y_{j} \leq C, \\
& y_{j} \in\{0,1\} \forall j \in B .
\end{aligned}
$$

Lower bounds for the Problem BLP-P are obtained in the algorithm by solving the following subproblem:

$$
\begin{aligned}
& (\mathbf{P P}-\mathbf{P}(\bar{y})) \quad q(\bar{y})=\min \sum_{j \in B} w_{j} \bar{y}_{j} r_{j}+\sum_{j \in N B} w_{j} r_{j}, \\
& r_{j}=\sum_{b \in \operatorname{ext}(P)} \mu_{b}^{j}, \quad j \in B \cup N B, \\
& x_{i}=\sum_{b \in \operatorname{ext}(P)} \mu_{b}^{j} b_{i}+f_{j i}, \quad j \in B \cup N B, i=1, \ldots, n, \\
& \mu_{b}^{j} \geq 0, \quad b \in \operatorname{ext}(P), j \in B \cup N B, \\
& r_{j} \geq 0, \quad j \in B \cup N B, \\
& x_{i} \in \mathbb{R}, \quad i=1, \ldots, n .
\end{aligned}
$$

If $\bar{r}$ is an optimal solution of the above problem for a given solution $\bar{y}$ feasible to the master problem $\mathbf{M P}$, the inequality $q \leq \sum_{j \in B} \sum_{i=1}^{n} w_{j} y_{j} \bar{r}_{j}+\sum_{j \in N B} \sum_{i=1}^{n} w_{j} \bar{r}_{j}$ either generates a new lower bound for MP or, if the optimal solution coincides with the previous one, it is a certificate of optimality. Based on this recursion, we propose the following algorithm:

Algorithm 1: Benders decomposition Algorithm

Initialization: Choose a solution $y^{0}$ satisfying the knapsack constraint, and solve the problem PP-P $(\bar{y})$ for $\bar{y}=y^{0}$. Let $r^{0}$ be an optimal solution for PP-P $(\bar{y})$. Take $\mathcal{P}=\{0\}$ and go to iteration $\nu=1$.

Iteration $\nu=1,2, \ldots$ : Solve the Master Problem MP. Let $y^{*}$ be an optimal solution of such problem and $q^{*}$ the corresponding optimal value.

- Solve PP-P $(\bar{y})$ for $\bar{y}=y^{*}$.If $q^{*}=q\left(y^{*}\right)$. END. 
- Otherwise, let $r^{*}$ be an optimal solution of PP-P $(\bar{y})$. Take $r^{\nu}=r^{*}$, $\mathcal{P}:=\mathcal{P} \cup\{\nu\}$, and go to iteration $\nu:=\nu+1$.

3.1.3. The case of the $\ell_{1}$-norm. In this section, we apply the above reasoning to the particular important case of problem BLP-P under the rectangular distance, that is, the distance induced by the $\ell_{1}$-norm. We take advantage of some specific properties of this norm to exploit further its algorithmic implications. As before, $n$ denotes the dimension of the space.

The set of extreme points of the unit ball of the $\ell_{1}$ norm is $\operatorname{ext}(P)=\left\{e_{1}, \ldots, e_{n},-e_{1}, \ldots,-e_{n}\right\}$, where $e_{i}$ the $i$-th vector of the canonical basis. Further, the $\ell_{1}$-norm of a vector $x$ is given by $\|x\|_{1}=\sum_{i=1}^{n}\left|x_{i}\right|$.

By introducing variables $r_{j i}$ representing the non linear terms $\left|x_{i}-f_{j i}\right|$, we adapt BLP-P to the $\ell_{1}$-norm case.

$\left(\mathbf{B L P}-\ell_{1}\right)$

$$
\begin{aligned}
& \max \sum_{j \in B} w_{j} y_{j} \sum_{i=1}^{n} r_{j i}+\sum_{j \in N B} w_{j} \sum_{i=1}^{n} r_{j i} \\
& \text { s.t. } \\
& \quad \sum_{j \in B} c_{j} y_{j} \leq C, \\
& \quad y_{j} \in\{0,1\}, \quad j \in B, \\
& \quad x \in \arg \min _{x} \sum_{j \in B} w_{j} y_{j} \sum_{i=1}^{n} r_{j i}+\sum_{j \in N B} w_{j} \sum_{i=1}^{n} r_{j i} \\
& \quad r_{j i} \geq x_{i}-f_{j i}, \quad j \in B \cup N B, i=1, \ldots, n, \\
& \quad r_{j i} \geq f_{j i}-x_{i}, \quad j \in B \cup N B, i=1, \ldots, n,
\end{aligned}
$$

As in Subsection 3.1.1, we can derive a MILP by using the primal dual optimality conditions and then linearizing the bilinear terms $y_{j} r_{j i}$ by introducing new variables $\hat{r}_{j i}$. In this formulation, dual variables $\alpha_{j i}$ correspond to contraints (16). The dual variables associated to constraints (17) have been eliminated. 
$\left(\mathrm{BLP}-\ell_{1}-\mathbf{1}\right)$

$$
\begin{aligned}
\max & \sum_{j \in B} w_{j} \sum_{i=1}^{n} \hat{r}_{j i}+\sum_{j \in N B} w_{j} \sum_{i=1}^{n} r_{j i} \\
\text { s.t. } & \sum_{j \in B} c_{j} y_{j} \leq C, \\
& y_{j} \in\{0,1\}, \quad j \in B, \\
& \sum_{j \in B} \sum_{i=1}^{n} w_{j} \hat{r}_{j i}+\sum_{j \in N B} \sum_{i=1}^{n} w_{j} r_{j i}=\sum_{j \in B \cup N B} \sum_{i=1}^{n}-f_{j i} \alpha_{j i}+ \\
& +\sum_{j \in B} \sum_{i=1}^{n} f_{j i}\left(w_{j} y_{j}-\alpha_{j i}\right)+\sum_{j \in N B} \sum_{i=1}^{n} f_{j i}\left(w_{j}-\alpha_{j i}\right), \\
& r_{j i} \geq x_{i}-f_{j i}, \quad j \in B \cup N B, i=1, \ldots, n, \\
& r_{j i} \geq f_{j i}-x_{i}, \quad j \in B \cup N B, i=1, \ldots, n, \\
& x_{i} \in \mathbb{R}, \quad i=1, \ldots, n, \\
& \hat{r}_{j i} \leq M_{j i} y_{j}, \quad j \in B, i=1, \ldots, n, \\
& \hat{r}_{j i} \leq r_{j i}, \quad j \in B, i=1, \ldots, n, \\
& \hat{r}_{j i} \geq r_{j i}-\left(1-y_{j}\right) M_{j i}, \quad j \in B, i=1, \ldots, n, \\
\hat{r}_{j i} \geq 0, \quad j \in B, i=1, \ldots, n, & \\
& \alpha_{j i} \leq w_{j} y_{j}, \quad j \in B, i=1, \ldots, n, \\
& \alpha_{j i} \leq w_{j}, \quad j \in B, i=1, \ldots, n, \\
& \alpha_{j i} \geq 0, \quad j \in B \cup N B, i=1, \ldots, n, \\
& \sum_{j \in B}\left(-2 \alpha_{j i}+w_{j} y_{j}\right)+\sum_{j \in N B}\left(-2 \alpha_{j i}+w_{j}\right)=0, \quad i=1, \ldots, n
\end{aligned}
$$

The reader can observe that valid big-M constant in this formulation are $M_{j i}=$ $\max _{k \in B \cup N B}\left|f_{k i}-f_{j i}\right|$, for all $i=1, \ldots, n$ and $j \in B$.

An alternative formulation can be derived for Problem BLP $-\ell_{1}$ by using the fact that the inner location problem can be decomposed into $n$ independent linear programs, one for each coordinate. Using the optimality conditions for each such problem and the linearization technique described above, we obtain a formulation 
$\mathbf{B L P}-\ell_{1} \mathbf{- 2}$ identical to $\mathbf{B L P}-\ell_{1} \mathbf{- 1}$ except that constraint (18) is replaced by the group of contraints:

$$
\sum_{j \in B} w_{j} \hat{r}_{j i}+\sum_{j \in N B} w_{j} r_{j i}=\sum_{j \in B \cup N B}-f_{j i} \alpha_{j i}+\sum_{j \in B} f_{j i}\left(w_{j} y_{j}-\alpha_{j i}\right)+\sum_{j \in N B} f_{j i}\left(w_{j}-\right.
$$
$\left.\alpha_{j i}\right), \quad i=1, \ldots, n$.

Algorithm 1 can also be adapted to the case of the $\ell_{1}$-norm. Then, $q(\bar{y})$ is obtained by solving the lower level problem defined by (15) - (17) but it can be solved in $O(n|B \cup N B|)$ time since, for each coordinate, it amounts to find the median of a discrete distribution.

We can also use the separability property in the proposed Benders Algorithm, by solving in each iteration $n$ subproblems $q_{i}(y)$ (one for each coordinate), and considering the following Master Problem:

$\left(\mathrm{MP}-\ell_{1}-\mathbf{i}\right)$

$$
\begin{array}{cl}
\max & \sum_{i=1}^{n} q_{i} \\
\text { s.t. } & \\
& q_{i} \leq \sum_{j \in B} w_{j} y_{j} z_{i j}^{\tau}+\sum_{j \in N B} w_{j} z_{i j}^{\tau} \quad \forall \tau \in \mathcal{P}, \quad \forall i=1, \ldots, n, \\
& \sum_{j \in B} c_{j} y_{j} \leq C, \\
& y_{j} \in\{0,1\} \forall j \in B,
\end{array}
$$

We will compare the performance of the four approaches in the computational study presented in Section 5 .

\subsection{Second approach: Evaluating the norm by its dual expression. Since} the polar set of a polyhedron is a polyhedron, $P^{o}$ induces the so-called dual norm of $\|\cdot\|_{P}$ that can also be used to evaluate $\|\cdot\|_{P}$. In this case, $\|\cdot\|_{P}$ is the optimal solution of the following linear program (see for example [20] or [22]):

$\left(\operatorname{NormP}^{0}\right)$

$$
\begin{aligned}
\|x\|_{P}= & \min r \\
\text { s.t. } & \sum_{i=1}^{n} u_{i} x_{i} \leq r, u \in \operatorname{ext}\left(P^{0}\right)
\end{aligned}
$$


Depending on the number and structure of the set of extreme points of $P$ and $P^{o}$, it may be more convenient to compute $\|\cdot\|_{P}$, by using its primal or dual expression. Fruther, this dual representation leads to different MILP formulations and the Benders approach can also be adapted.

3.2.1. MILP formulation: Using the dual representation of the norm, BLP can be formulated as:

$\left(\mathrm{BLP}^{\mathrm{P}} \mathbf{P}^{0}\right.$

$$
\begin{aligned}
\max & \sum_{j \in B} w_{j} y_{j} r_{j}+\sum_{j \in N B} w_{j} r_{j} \\
\text { s.t. } & \sum_{j \in B} c_{j} y_{j} \leq C, \\
& y_{j} \in\{0,1\} \quad j \in B, \\
& r \in \arg \min _{x, r} \sum_{j \in B} w_{j} y_{j} r_{j}+\sum_{j \in N B} w_{j} r_{j}, \\
& r_{j} \geq \sum_{i=1}^{n} u_{i}\left(x_{i}-f_{j i}\right), \quad u \in \operatorname{ext}\left(P^{0}\right), j \in B \cup N B, \\
& r_{j} \geq 0, \quad j \in B \cup N B, \\
& x_{i} \in \mathbb{R}, \quad i=1, \ldots, n,
\end{aligned}
$$

where variables $r_{j}$, defined in constraint (29), represent the distance between $x$ and $f_{j}$. Constraints (1) and (2) relate the choice of the location of the primary facilities, according to a budget constraint, and constraints (9), (29), (7) and (8) define the inner subproblem, in which the representation of the norm via its dual expression has been included.

Proposition 2. Problem $\boldsymbol{B L P}-\boldsymbol{P}^{0}$ can be formulated as the following single level problem $\boldsymbol{B L P}-\boldsymbol{P}^{0}$.

$$
\begin{gathered}
\left(\mathbf{B L P}-\mathbf{P}^{0}\right)^{\prime} \quad \max \sum_{j \in B} w_{j} y_{j} r_{j}+\sum_{j \in N B} w_{j} r_{j} \\
\text { s.t. } \sum_{j \in B} c_{j} y_{j} \leq C,
\end{gathered}
$$




$$
\begin{aligned}
& y_{j} \in\{0,1\} \quad j \in B, \\
& \sum_{j \in B} w_{j} y_{j} r_{j}+\sum_{j \in N B} w_{j} r_{j}=\sum_{u \in \operatorname{ext}\left(P^{0}\right)} \sum_{j \in B \cup N B}\left(\sum_{i=1}^{n}-f_{j i} u_{i}\right) \gamma_{u j}, \\
& r_{j} \geq \sum_{i=1}^{n} u_{i}\left(x_{i}-f_{j i}\right), \quad u \in \operatorname{ext}\left(P^{0}\right), j \in B \cup N B, \\
& r_{j} \geq 0, \quad j \in B \cup N B, \\
& x_{i} \in \mathbb{R}, \quad i=1, \ldots, n, \\
& \sum_{u \in \operatorname{ext}\left(P^{0}\right)} \gamma_{u j} \leq w_{j} y_{j}, \quad j \in B, \\
& \sum_{u \in \operatorname{ext}\left(P^{0}\right)} \gamma_{u j} \leq w_{j}, \quad j \in N B, \\
& \sum_{u \in \operatorname{ext}\left(P^{0}\right)} \sum_{j \in B \cup N B}\left(-u_{i}\right) \gamma_{u j}=0, \quad i=1, \ldots n, \\
& \gamma_{u j} \geq 0 \quad u \in \operatorname{ext}\left(P^{0}\right), j \in B \cup N B
\end{aligned}
$$

The proof of this proposition follows the same lines as that of Proposition 1 and is thus omitted.

We can observe that in the above formulation there appear the same bilinear terms that we have already obtained in Section 3.1.1. Therefore, the same linearization (14) can be applied to obtain the corresponding MILP formulation.

3.2.2. Benders like algorithm for solving $\boldsymbol{B L} \boldsymbol{P}-\boldsymbol{P}^{0}$. The Benders Algorithm proposed in Section 3.1.2 can also be applied when the norm is induced by the polar polyhedron, with the same Master Problem MP and the following primal problem:

$\left(\mathbf{P P}-\mathbf{P}^{0}\right)$

$$
\begin{array}{ll}
\min & q(y)=\sum_{j \in B} w_{j} y_{j} r_{j}+\sum_{j \in N B} w_{j} r_{j}, \\
\text { s.t. } & r_{j} \geq \sum_{i=1}^{n} u_{k i}\left(x_{i}-f_{j i}\right), \quad u \in \operatorname{ext}\left(P^{0}\right), j \in B \cup N B, \\
& r \geq 0, x \in \mathbb{R}^{n}, \\
& x_{i} \in \mathbb{R}, \quad i=1, \ldots, n .
\end{array}
$$


3.2.3. The case of the $\ell_{\infty}$-norm. This is Section, we apply the above results to the important case of the infinity norm. The set of extreme points of the infinity norm is $\operatorname{ext}(P)=\left\{\left(a_{1}, \ldots, a_{n}\right) \in \mathbb{R}^{n}: a_{i} \in\{1,-1\}, i=1, . . n,\right\}$, so that $|\operatorname{ext}(P)|=2^{n}$. Then formulation BLP-P' would include $2^{n}(|B|+|N B|) \mu_{e}^{j}$ variables, and more than $2^{n}(|B|+|N B|)$ constraints. However, the number of extreme points of the polar polyhedron is much smaller: $\operatorname{ext}\left(P^{0}\right)=\left\{e_{1}, \ldots, e_{n},-e_{1}, \ldots,-e_{n}\right\}$ and $\left|\operatorname{ext}\left(P^{0}\right)\right|=2 n$. Further, the $\ell_{\infty}$-norm of a vector $x$ is given by $\|x\|_{\infty}=\max _{i=1, \ldots, n}\left|x_{i}\right|$. This allows to adapt BLP-P to the $\ell_{\infty}$-norm case as follows:

$$
\begin{aligned}
\left(\mathbf{B L P}-\ell_{\infty}\right) \quad & \max \\
& \sum_{j \in B} w_{j} y_{j} r_{j}+\sum_{j \in N B} w_{j} r_{j} \\
\text { s.t. } & \sum_{j \in B} c_{j} y_{j} \leq C, \\
& y_{j} \in\{0,1\}, \quad j \in B, \\
& x \in \arg \min _{x} \sum_{j \in B} w_{j} y_{j} r_{j}+\sum_{j \in N B} w_{j} r_{j}, \\
& r_{j} \geq x_{i}-f_{j i}, \quad j \in B \cup N B, i=1, \ldots, n, \\
& r_{j} \geq f_{j i}-x_{i}, \quad j \in B \cup N B, i=1, \ldots, n .
\end{aligned}
$$

Further, a MILP formulation can also be derived.

$$
\begin{aligned}
\left(\mathbf{B L P}-\ell_{\infty}-\mathbf{1}\right) \quad \max & \sum_{j \in B} w_{j} \hat{r}_{j}+\sum_{j \in N B} w_{j} r_{j} \\
\text { s.t. } & \sum_{j \in B} c_{j} y_{j} \leq C, \\
& y_{j} \in\{0,1\}, \quad j \in B, \\
& \sum_{j \in B} w_{j} \hat{r}_{j}+\sum_{j \in N B} w_{j} r_{j}=\sum_{i=1}^{n} \sum_{j \in B \cup N B} f_{j i}\left(-\gamma_{e_{i} j}+\gamma_{-e_{i} j}\right), \\
& r_{j} \geq x_{i}-f_{j i}, \quad j \in B \cup N B, i=1, \ldots, n, \\
& r_{j} \geq f_{j i}-x_{i}, \quad j \in B \cup N B, i=1, \ldots, n,
\end{aligned}
$$




$$
\begin{aligned}
& r_{j} \geq 0, \quad j \in B \cup N B, \\
& \sum_{i=1}^{n}\left(\gamma_{e_{i} j}+\gamma_{-e_{i} j}\right) \leq w_{j} y_{j}, \quad j \in B, \\
& \sum_{i=1}^{n}\left(\gamma_{e_{i} j}+\gamma_{-e_{i} j}\right) \leq w_{j}, \quad j \in N B, \\
& \sum_{j \in B \cup N B}\left(-\gamma_{e_{i} j}+\gamma_{-e_{i} j}\right)=0, \quad i=1, \ldots, n, \\
& \gamma_{e_{i} j} \geq 0, \quad j \in B \cup N B, i=1, \ldots, n, \\
& \gamma_{-e_{i} j} \geq 0, \quad j \in B \cup N B, i=1, \ldots, n, \\
& \hat{r}_{j} \leq M y_{j}, \quad j \in B \\
& \hat{r}_{j} \leq r_{j}, \quad j \in B, \\
& \hat{r}_{j} \geq r_{j}-\left(1-y_{j}\right) M, \quad j \in B, \\
& \hat{r}_{j} \geq 0, \quad j \in B .
\end{aligned}
$$

Finally, The proposed Benders algorithm can also be applied to the problem under the $\ell_{\infty}$ norm. The resulting inner subproblem is given by the lower level problem of $\mathrm{BLP}-\ell_{\infty}$.

\section{EXTENSIONS}

This section is devoted to present extensions of the problem BLP to several secondary facilities and non-polyhedral norms. We analyze the problem with $K>1$ secondary facilities which means to locate $K$ new facilities also in the lower level problem. Moreover, we extend the problem BLP to deal with norms $\ell_{\tau}$ for $\tau \in \mathbb{Q}$, $\tau \geq 1$ which requires to apply conic programming and conic duality to obtain results similar to the ones presented in previous sections.

\subsection{The model with $K$ secondary facilities (independent followers) - We} are interested to incorporate to the problem BLP the possibility to locate several secondary facilities rather than only one, and the goal of each secondary facility is to minimize the overall distance to the primary facilities. In the following, we analyze 
problem BLP with $K$ secondary points to be located in the lower level problem, that is, we consider that instead of locating one secondary facility, $K$ of these points must be located. For this extension we assume that we are given vectors of weights $w^{k} \in \mathbb{R}_{+}^{n}$, for $k=1, \ldots, K$, and we define $K$ vectors of decision variables $x^{k} \in \mathbb{R}^{n}$, for $k=1, \ldots, K$; where $x^{k}$ are the coordinates of the location of the $k$-th secondary point. With this notation, the new problem can be written as:

$$
\begin{aligned}
\text { (BLP-K }) \quad \max & \sum_{k \in K}\left(\sum_{j \in B} w_{j}^{k} d\left(x^{k}, f_{j}\right) y_{j}+\sum_{j \in N B} w_{j}^{k} d\left(x^{k}, f_{j}\right)\right) \\
\text { s.t. } & \sum_{j \in B} c_{j} y_{j} \leq C, \\
& y_{j} \in\{0,1\}, \quad j \in B, \\
& x^{k} \in \arg \min _{x^{k}} \sum_{j \in B} w_{j}^{k} d\left(x^{k}, f_{j}\right) y_{j}+\sum_{j \in N B} w_{j}^{k} d\left(x^{k}, f_{j}\right) \quad \forall k=1, \ldots, K .
\end{aligned}
$$

In the particular case in which $w^{1}=w^{2}=\ldots=w^{k}$, we observe that by symmetry, there is an optimal solution where the secondary facilities co-locate.

Coming back to the general problem BLP-K, the evaluation of the norm can be done via the primal or dual expression. In both cases, in order to develop a MILP formulation for the model with $K$ secondary facilities, we can apply the same technique that in the previous section. Given a solution $y$ of the upper level problem, the continuous location problem of each follower is linear and thus, the strong duality theorem can be applied as before. This implies that $K$ different one-secondary facility problems are added to the leader problem. In conclusion, the same approach used with the one-secondary facility location problem is replicated $K$ times and the same results follow.

Furthermore, the Benders algorithm can also be extended to the case with $K$ followers. The Master Problem for this extension must be slightly modified:

$(\mathbf{M P}-\mathbf{K}) \quad \max \sum_{k \in K} q^{k}$ 


$$
\begin{array}{ll}
\text { s.t. } & q^{k} \leq \sum_{j \in B} \sum_{i=1}^{n} w_{j}^{k} y_{j} r_{j}^{k \tau}+\sum_{j \in N B} \sum_{i=1}^{n} w_{j}^{k} r_{j}^{k \tau} \quad \forall \tau \in \mathcal{P}, \forall k \in K, \\
& \sum_{j \in B} c_{j} y_{j} \leq C, \\
& y_{j} \in\{0,1\} \forall j \in B .
\end{array}
$$

In addition, in this formulation, there are $K$ primal subproblems with the same structure but with different set of $w$ weights. Therefore, in each iteration of this Benders approach, $K$ primal subproblems must be solved.

4.2. The problem under the $\ell_{\tau}$-norm . This section extends the analysis of the problem to the case where the inner subproblem measures distances with $\ell_{\tau}$-norms with $\tau \in \mathbb{Q}, \tau \geq 1$. Recall that $\|x\|_{\tau}=\left(\sum_{i=1}^{n}\left|x_{i}\right|^{\tau}\right)^{1 / \tau}$.

The problem to be considered in this case is

$\left(\mathrm{BLP}-\ell_{\tau}\right)$

$$
\begin{aligned}
\max & \sum_{j \in B} w_{j}\left\|x-f_{j}\right\|_{\tau} y_{j}+\sum_{j \in N B} w_{j}\left\|x-f_{j}\right\|_{\tau} \\
\text { st. } & \sum_{j \in B} c_{j} y_{j} \leq C \\
& y_{j} \in\{0,1\} \quad j \in B, \\
& x \in \arg \min _{x} \sum_{j \in B} w_{j}\left\|x-f_{j}\right\|_{\tau} y_{j}+\sum_{j \in N B} w_{j}\left\|x-f_{j}\right\|_{\tau},
\end{aligned}
$$

Let $\rho \in \mathbb{Q}$ be such that $1 / \tau+1 / \rho=1$.

In order to reformulate Problem BLP- $\ell_{\tau}$ we give the following proposition, using the representation given in Blanco et al [3].

Proposition 3. The problem $\boldsymbol{B L P}-\ell_{\tau}$ can be reformulated as a the single level mixed integer conic program.

$(\mathbf{C o n i c P}) \quad \max \sum_{j \in B} w_{j}\left\|x-f_{j}\right\|_{\tau} y_{j}+\sum_{j \in N B} w_{j}\left\|x-f_{j}\right\|_{\tau}$ 


$$
\begin{array}{ll}
\text { s.t. } & \sum_{j \in B} c_{j} y_{j} \leq C, \\
& y_{j} \in\{0,1\} \quad j \in B, \\
& \sum_{j \in B} w_{j}\left\|x-f_{j}\right\|_{\tau} y_{j}+\sum_{j \in N B} w_{j}\left\|x-f_{j}\right\|_{\tau}=\sum_{j \in B \cup N B} \sum_{i=1}^{n} V_{j i} f_{j i}, \\
& x^{+}-x^{-}-Z_{j}=f_{j}, \quad \forall j \in B \cup N B, \\
& \left\|Z_{j}\right\|_{\tau} \leq r_{j}, \quad \forall j \in B \cup N B, \\
& x^{+}, x^{-} \in \mathbb{R}_{+}^{n}, Z_{j} \in \mathbb{R}^{n}, r \in \mathbb{R}^{n}, \\
& \sum_{j \in B \cup N B} V_{j i}+\lambda_{i}^{1}=0, \quad \forall i=1, \ldots, n, \\
& -\sum_{j \in B \cup N B} V_{j i}+\lambda_{i}^{2}=0, \quad \forall i=1, \ldots, n, \\
& -V_{j i}+\mu_{j i}=0, \quad \forall j \in B \cup N B, i=1, \ldots, n, \\
& \left\|\mu_{j}\right\|_{\rho} \leq \gamma_{j}, \quad \forall j \in B \cup N B, \\
& \gamma_{j}=w_{j} y_{j}, \quad \text { if } i \in B, \\
\gamma_{j}=w_{j}, \quad \text { if } i \in N B, & \lambda^{1}, \lambda^{2} \in \mathbb{R}_{+}^{n}, \mu_{j} \in \mathbb{R}^{n}, \forall j \in B \cup N B, \gamma \in \mathbb{R}^{n} .
\end{array}
$$

Proof. We observe that the inner location problem can be formulated as the following conic linear program in standard form [16]:

$$
\begin{array}{ll}
\min & \sum_{j \in B} w_{j} y_{j} r_{j}+\sum_{j \in N B} w_{j} r_{j} \\
\text { s.t. } & x^{+}-x^{-}-Z_{j}=f_{j}, \quad \forall j \in B \cup N B, \\
& \left\|Z_{j}\right\|_{\tau} \leq r_{j}, \quad \forall j \in B \cup N B, \\
& x^{+}, x^{-} \in \mathbb{R}_{+}^{n}, Z_{j} \in \mathbb{R}^{n}, r \in \mathbb{R}^{n} .
\end{array}
$$

Therefore, its conic dual can be written in the following form:

$$
\max \sum_{j \in B \cup N B} \sum_{i=1}^{n} V_{j i} f_{j i}
$$




$$
\begin{array}{ll}
\text { s.t. } & \sum_{j \in B \cup N B} V_{j i}+\lambda_{i}^{1}=0, \quad \forall i=1, \ldots, n,, \\
& -\sum_{j \in B \cup N B} V_{j i}+\lambda_{i}^{2}=0, \quad \forall i=1, \ldots, n, \\
& -V_{j i}+\mu_{j i}=0, \quad \forall j \in B \cup N B, i=1, \ldots, n, \\
& \left\|\mu_{j}\right\|_{\rho} \leq \gamma_{j}, \quad \forall j \in B \cup N B, \\
& \gamma_{j}=w_{j} y_{j}, \text { if } j \in B, \\
& \gamma_{j}=w_{j}, \text { if } j \in N B, \\
& \lambda^{1}, \lambda^{2} \in \mathbb{R}_{+}^{n}, \mu_{j} \in \mathbb{R}^{n}, \forall j \in B \cup N B, \gamma \in \mathbb{R}^{n} .
\end{array}
$$

Clearly, the inner primal and dual problems satisfy Slater condition so that strong duality applies. This allows us to insert the optimality conditions in BLP- $\ell_{\tau}$ to obtain the final single level program ConicP.

\section{Computational Results}

In the following we report some numerical results conducted to compare the efficiency of the different methods proposed to solve BLP, and to check experimentally their scope.

The computational experiments were carried out on a personal computer with Intel B. Core (TM) i7-4720HQ, 2.60 gigahertz with 16384 megabytes RAM. The MILP formulations and algorithms were implemented and solved by using Xpress Version 8.0.

The distances considered for the numerical experiments were computed using the $\ell_{1}$ and $\ell_{\infty}$ norms. Therefore, we implemented the MILP formulations and algorithms proposed in Sections 3.1.3 and 3.2.3, in which we adapted the general methods in Section 3 to the models with these two particular distances.

For the computational study we generated different random instances taking into account the following factors: the dimension of the space, $n$, the cardinality of $B$ and $N B$, which are the set of potential locations for the new primary facilities and the set of existing primary facilities, respectively, and also the maximum budget $C$. We considered the following levels for each factor: 
- $n=2,3,10,20$,

- $|B|=1000,2000,5000,10000$,

- $|N B|=\frac{1}{4}|B|, \frac{1}{3}|B|, \frac{1}{2}|B|$,

- $C=\frac{1}{C^{\prime}}|B|=\frac{1}{4}|B|, \frac{1}{3}|B|$.

The weights $w_{j}$ were generated randomly in the interval $[0,1]$, and each coordinate, $f_{j i}$, of the location of the primary facilities $f_{j}$ was generated randomly in the interval $[-1000,1000]$, for all the instances.

For each combination of levels, 5 different instances were generated and solved. The CPU time limit to solve the problems was set to 1800 seconds.

In Figures 1 and 2 we show the performance profile graphs of the number of solved instances for the different proposed models for the $\ell_{1}$-norm (Figure 1) and $\ell_{\infty}$-norm (Figure 2). We represent in the abscissa axis the time (in seconds) and in the ordinate axis the number of solved instances. Figure 1 reports the results for the $\ell_{1}$-norm and it compares the two MILP formulations BLP- $\ell_{1}-\mathbf{1}$ and BLP- $\ell_{1}-\mathbf{2}$, the basic Benders algorithm, that we denote by Bend $\ell_{1}$, and the Benders algorithm using the separability property, denoted as Bend- $\ell_{1}$-sep. Figure 2 shows the results for the $\ell_{\infty}$-norm and it compares the MILP formulation, BLP- $\ell_{\infty} \mathbf{- 1}$, and Benders algorithm, denoted as Bend- $\ell_{\infty}$.

We can observe in Figures 1 and 2 that the Benders algorithms are more efficient than the MILP formulations, in both cases with the $\ell_{1}$-and- $\ell_{\infty}$-norm cases. The Benders algorithms solve all the instances in very short time, whereas none of the MILP formulations could solve to optimality all the instances. We can see in the figures that the formulations BLP- $\ell_{1}-\mathbf{1}$ and $\mathbf{B L P}-\ell_{1}-\mathbf{2}$ solve around 300 out of the 480 instances in 1800 seconds, and formulation BLP- $\ell_{\infty} \mathbf{- 1}$ solves around 400 instances in the same time.

The average number of cuts added in the Benders algorithm is 5,03 for Bend- $\ell_{1}$, 4,75 for Bend- $\ell_{1}$-sep and 4,28 for Bend- $\ell_{\infty}$. The maximum number of Benders cuts, 14 , was added for the Bend- $\ell_{1}$-sep for an instance with $n=10,|B|=5000$, $|N B|=6667$ and $C=\frac{1}{4}|B|$.

For the $\ell_{1}$-norm case, in Figure 1, we can see that the Benders algorithm Bend- $\ell_{1}$ solves all the instances in approximately 200 seconds, whereas the Benders algorithm 
using the separability property, Bend- $\ell_{1}$-sep, needs a bit more time. Nevertheless the performance of both methods is very similar. The same trend can be observed for the MILP formulations, the one without the separability property could solve in the end more instances within the same time limit. However, BLP- $\ell_{1}-\mathbf{2}$ works better for the big instances, $n=10,20$ (see Figure 3 in the Appendix).

With respect to the $\ell_{\infty}$-norm case, Figure 2 shows that the Benders algorithm solves all the instances in less than 51 seconds, meanwhile BLP- $\ell_{\infty} \mathbf{- 1}$ only solves, in the same time, approximately one half of the instances.

More details about the Computational Results can be found in Tables 1 and 2 in the Appendix. We report the average CPU times (CPU), and the numbers of problems, out of 5, solved to optimality (\#OPT), for each type of instance and each formulation or algorithm.

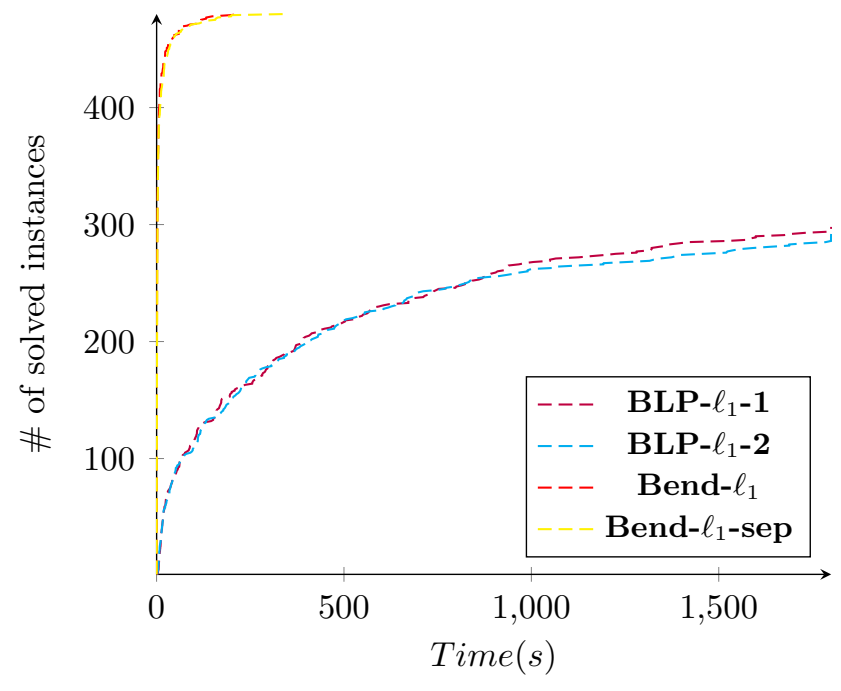

Figure 1. Performance profile graph of \#solved instances for the different proposed models for the $\ell_{1}$ norm.

\section{Conclusions}

This paper considers models for the location of controversial facilities. Controversial facilities must be understood as those facilities such that their placement induces a disagreement among users with different, non-aligned or opposite interests. Semiobnoxious facility location and the location and protection of critical infrastructures 


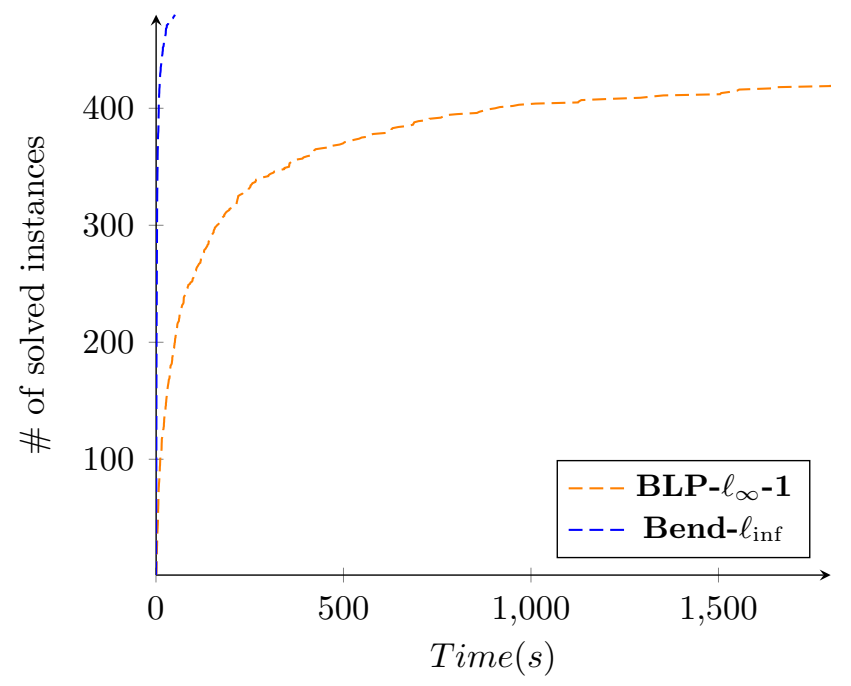

Figure 2. Performance profile graph of \#solved instances for the different proposed models for the $\ell_{\infty}$ norm.

or facilities sensitive to intentional attacks are typical examples of this area of research.

We model these situations by a bilevel optimization problem. The first level locates primary facilities trying to be as far away as possible from the secondary ones, which in turns, wish to be as close as possible to the primary ones. We develop mathematical programming formulations for the above mentioned bilevel programs as well as some algorithms that perform very-well in all our experiments that range from small problems on the plane $(\mathrm{n}=2)$ with up to $|B|=10000$, possibilities for the primary facilities until dimension $n=20$ and $|B|=10000$.

\section{ACKNOWLEDGEMENTS}

Martine Labbé has been partially supported by the Fonds de la Recherche Scientique - FNRS under Grant(s) no PDR T0098.18. The last two authors have been partially supported by MINECO Spanish/FEDER grants number MTM2016-74983C02-01.

\section{Appendix}




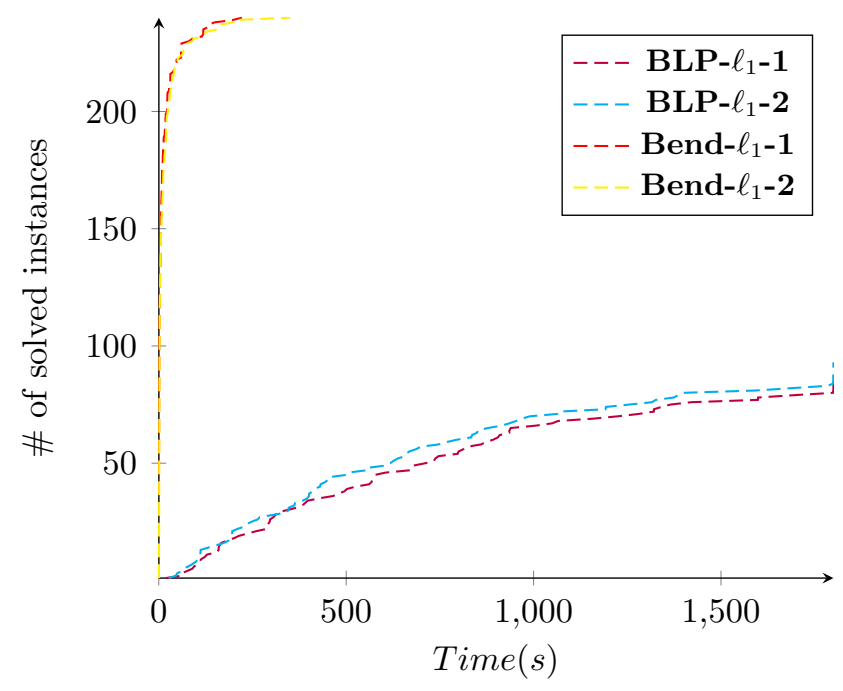

FiguRE 3. Performance profile graph of \#solved instances for the different proposed models for big instances $(n=10,20)$ for the $\ell_{1}$ norm. 


\begin{tabular}{|c|c|c|c|c|c|c|c|c|c|c|c|c|c|c|c|}
\hline \multicolumn{4}{|c|}{ INSTANCES } & \multicolumn{2}{|c|}{ BLP- $\ell_{1}-1$} & \multicolumn{2}{|c|}{ BLP- $\ell_{1}-2$} & \multicolumn{2}{|c|}{ Alg- $\ell_{1}$} & \multicolumn{2}{|c|}{ Alg- $\ell_{1}$-sep } & \multicolumn{2}{|c|}{$\mathrm{BLP}-\ell_{\infty}-1$} & \multicolumn{2}{|c|}{$\mathrm{Alg}-\ell_{\infty}$} \\
\hline $\mathrm{n}$ & $|\mathbf{B}|$ & $|\mathbf{N B}|$ & $\mathrm{C}^{\prime}$ & \#OPT & CPU & \#OPT & CPU & \#OPT & CPU & \#OPT & CPU & \#OPT & CPU & \#OPT & CPU \\
\hline$\overline{2}$ & 1000 & $\overline{250}$ & 3 & 5 & 10,82 & 5 & $\overline{10,36}$ & 5 & $\overline{0,41}$ & 5 & $\overline{0,50}$ & 5 & $\overline{2,13}$ & $\overline{5}$ & $\overline{0,56}$ \\
\hline 2 & 1000 & 250 & 4 & 5 & 4,80 & 5 & 5,89 & 5 & 0,62 & 5 & 0,52 & 5 & 2,22 & 5 & 1,03 \\
\hline 2 & 1000 & 333 & 3 & 5 & 13,39 & 5 & 10,07 & 5 & 0,35 & 5 & 0,41 & 5 & 2,19 & 5 & 0,65 \\
\hline 2 & 1000 & 333 & 4 & 5 & 7.57 & 5 & 4,98 & 5 & 0,55 & 5 & 0,62 & 5 & 2,47 & 5 & 1,05 \\
\hline 2 & 1000 & 500 & 3 & 5 & 11,70 & 5 & 12,76 & 5 & 0,38 & 5 & 0,43 & 5 & 8,53 & 5 & 0,81 \\
\hline 2 & 1000 & 500 & 4 & 5 & 7,86 & 5 & 6,35 & 5 & 0,54 & 5 & 0,52 & 5 & 3,02 & 5 & 0,95 \\
\hline 2 & 2000 & 500 & 3 & 5 & 36,59 & 5 & 37,27 & 5 & 0,75 & 5 & 1,44 & 5 & 6,34 & 5 & 0,79 \\
\hline 2 & 2000 & 500 & 4 & 5 & 13,36 & 5 & 11,66 & 5 & 0,65 & 5 & 0,73 & 5 & 9,15 & 5 & 1,36 \\
\hline 2 & 2000 & 667 & 3 & 5 & 217,01 & 5 & 152,79 & 5 & 0,68 & 5 & 0,85 & 5 & 7,20 & 5 & 0,92 \\
\hline 2 & 2000 & 667 & 4 & 5 & 20,58 & 5 & 14,54 & 5 & 0,45 & 5 & 0,60 & 5 & 6,14 & 5 & 1,17 \\
\hline 2 & 2000 & 1000 & 3 & 5 & 56,48 & 5 & 78,63 & 5 & 0,45 & 5 & 0,61 & 5 & 15,29 & 5 & 0,81 \\
\hline 2 & 2000 & 1000 & 4 & 5 & 29,85 & 5 & 20,60 & 5 & 0,52 & 5 & 0,65 & 5 & 12,74 & 5 & 1,56 \\
\hline 2 & 5000 & 1250 & 3 & 5 & 204,62 & 5 & 508,34 & 5 & 1,19 & 5 & 1,58 & 5 & 48,51 & 5 & 1,62 \\
\hline 2 & 5000 & 1250 & 4 & 5 & 124,68 & 5 & 97,10 & 5 & 1,49 & 5 & 2,36 & 5 & 52,24 & 5 & 1,70 \\
\hline 2 & 5000 & 1667 & 3 & 5 & 381,53 & 5 & 268,18 & 5 & 1,03 & 5 & 1,32 & 5 & 45,06 & 5 & 1,47 \\
\hline 2 & 5000 & 1667 & 4 & 5 & 135,94 & 5 & 198,89 & 5 & 2,08 & 5 & 1,66 & 5 & 37,01 & 5 & 1,96 \\
\hline 2 & 5000 & 2500 & 3 & 5 & 416.01 & 5 & 369,43 & 5 & 0,80 & 5 & 1,12 & 5 & 139,15 & 5 & 2,34 \\
\hline 2 & 5000 & 2500 & 4 & 5 & 107,17 & 5 & 405,78 & 5 & 1,23 & 5 & 1,89 & 5 & 38,32 & 5 & 1,92 \\
\hline 2 & 10000 & 2500 & 3 & 4 & 708,80 & 3 & 955,21 & 5 & 1,68 & 5 & 2,57 & 5 & 137,97 & 5 & 3,29 \\
\hline 2 & 10000 & 2500 & 4 & 5 & 325,03 & 5 & 355,51 & 5 & 2,44 & 5 & 3,75 & 5 & 81,66 & 5 & 3,72 \\
\hline 2 & 10000 & 3333 & 3 & 5 & 390,82 & 5 & 296,18 & 5 & 1,94 & 5 & 2,78 & 5 & 121,14 & 5 & 3,42 \\
\hline 2 & 10000 & 3333 & 4 & 5 & 329,09 & 5 & 439,71 & 5 & 3,09 & 5 & 3,46 & 5 & 159,98 & 5 & 3,16 \\
\hline 2 & 10000 & 5000 & 3 & 5 & 506,03 & 4 & 671,22 & 5 & 2,16 & 5 & 2,09 & 4 & 530,49 & 5 & 3,38 \\
\hline 2 & 10000 & 5000 & 4 & 5 & 516,73 & 5 & 416,86 & 5 & 2,83 & 5 & 3,27 & 5 & 141,33 & 5 & 3,77 \\
\hline 3 & 1000 & 250 & 3 & 5 & 9,67 & 5 & 19,03 & 5 & 0,21 & 5 & 0,27 & 5 & 5,95 & 5 & 0,70 \\
\hline 3 & 1000 & 250 & 4 & 5 & 58,74 & 5 & 43,20 & 5 & 0,63 & 5 & 0,71 & 5 & 3,09 & 5 & 1,18 \\
\hline 3 & 1000 & 333 & 3 & 5 & 21,51 & 5 & 50,24 & 5 & 0,36 & 5 & 0,37 & 5 & 6,99 & 5 & 0,60 \\
\hline 3 & 1000 & 333 & 4 & 5 & 29,05 & 5 & 32,52 & 5 & 0.65 & 5 & 0,90 & 5 & 4,68 & 5 & 1,21 \\
\hline 3 & 1000 & 500 & 3 & 5 & 26,14 & 5 & 28,70 & 5 & 0,34 & 5 & 0,49 & 5 & 10,17 & 5 & 0,57 \\
\hline 3 & 1000 & 500 & 4 & 5 & 33,46 & 5 & 47,28 & 5 & 0.38 & 5 & 0,52 & 5 & 6,86 & 5 & 0.73 \\
\hline 3 & 2000 & 500 & 3 & 5 & 120,41 & 5 & 144,41 & 5 & 0,38 & 5 & 0,53 & 5 & 63,77 & 5 & 1,19 \\
\hline 3 & 2000 & 500 & 4 & 5 & 389,05 & 5 & 301,49 & 5 & 0.76 & 5 & 1,22 & 5 & 21,33 & 5 & 1,41 \\
\hline 3 & 2000 & 667 & 3 & 5 & 316,33 & 5 & 232,75 & 5 & 1,04 & 5 & 0,92 & 5 & 31,18 & 5 & 1,04 \\
\hline 3 & 2000 & 667 & 4 & 5 & 129,52 & 5 & 224,55 & 5 & 0,47 & 5 & 0,56 & 5 & 21,81 & 5 & 1,48 \\
\hline 3 & 2000 & 1000 & 3 & 5 & 113,90 & 5 & 261,64 & 5 & 0,65 & 5 & 0,53 & 5 & 81,42 & 5 & 1,38 \\
\hline 3 & 2000 & 1000 & 4 & 5 & 179,31 & 5 & 249,42 & 5 & 0,62 & 5 & 0,71 & 5 & 11,05 & 5 & 0,88 \\
\hline 3 & 5000 & 1250 & 3 & 4 & 1014,05 & 3 & 1310,24 & 5 & 1,95 & 5 & 1,85 & 5 & 131,33 & 5 & 1,94 \\
\hline 3 & 5000 & 1250 & 4 & 3 & 1352,73 & 2 & 1442,08 & 5 & 2,43 & 5 & 2,24 & 5 & 106,38 & 5 & 2,12 \\
\hline 3 & 5000 & 1667 & 3 & 4 & 763,66 & 4 & 669,57 & 5 & 1,14 & 5 & 1,28 & 5 & 180,78 & 5 & 1,94 \\
\hline 3 & 5000 & 1667 & 4 & 3 & 1102,02 & 4 & 1047,36 & 5 & 1,56 & 5 & 1,91 & 5 & 213,59 & 5 & 2,08 \\
\hline 3 & 5000 & 2500 & 3 & 5 & 636,19 & 5 & 689,33 & 5 & 0,76 & 5 & 1,14 & 5 & 391,97 & 5 & 2,07 \\
\hline 3 & 5000 & 2500 & 4 & 5 & 592,53 & 2 & 1223,05 & 5 & 1,42 & 5 & 1,58 & 5 & 77,89 & 5 & 1,90 \\
\hline 3 & 10000 & 2500 & 3 & 1 & 1778,05 & 1 & 1800,00 & 5 & 2,42 & 5 & 3,38 & 5 & 899,86 & 5 & 3,09 \\
\hline 3 & 10000 & 2500 & 4 & 1 & 1727,79 & 0 & - & 5 & 5,59 & 5 & 5,16 & 5 & 198,48 & 5 & 4,18 \\
\hline 3 & 10000 & 3333 & 3 & 3 & 1127,45 & 1 & 1732,91 & 5 & 2,67 & 5 & 3,91 & 5 & 832,12 & 5 & 3,17 \\
\hline 3 & 10000 & 3333 & 4 & 3 & 1253,69 & 2 & 1321,56 & 5 & 4,79 & 5 & 6,15 & 4 & 797,61 & 5 & 3,91 \\
\hline 3 & 10000 & 5000 & 3 & 3 & 1379,12 & 1 & 1771,16 & 5 & 2,86 & 5 & 4,41 & 4 & 470,05 & 5 & 4,01 \\
\hline 3 & 10000 & 5000 & 4 & 0 & $\begin{array}{ll}- & \\
-\end{array}$ & 0 & - & 5 & 4,18 & 5 & 5,79 & 5 & 325,39 & 5 & 4,87 \\
\hline
\end{tabular}

TABLE 1. Numerical results for BLP under the $l_{1}$ and $e l l_{\infty}$ norm. 


\begin{tabular}{|c|c|c|c|c|c|c|c|c|c|c|c|c|c|c|c|}
\hline \multicolumn{4}{|c|}{ INSTANCES } & \multicolumn{2}{|c|}{ BLP- $\ell_{1}-1$} & \multicolumn{2}{|c|}{ BLP- $\ell_{1}-2$} & \multicolumn{2}{|c|}{ Alg- $\ell_{1}$} & \multicolumn{2}{|c|}{ Alg- $\ell_{1}$-sep. } & \multicolumn{2}{|c|}{ BLP- $\ell_{\infty}-1$} & \multicolumn{2}{|c|}{ Alg- $\ell_{\infty}$} \\
\hline $\mathrm{n}$ & $|\mathbf{B}|$ & $|\mathbf{N B}|$ & $\mathrm{C}$ & \#OPT & CPU & \#OPT & CPU & \#OPT & CPU & \#ОРТ & $\mathrm{CPU}$ & \#OPT & $\mathrm{CPU}$ & \#OPT & $\mathrm{CPU}$ \\
\hline 10 & 1000 & 250 & 3 & 5 & 175,69 & 5 & 262,45 & 5 & 0,52 & 5 & $\overline{0,84}$ & 5 & 23,53 & 5 & $\overline{0,73}$ \\
\hline 10 & 1000 & 250 & 4 & 5 & 286,71 & 5 & 199,67 & 5 & 0,58 & 5 & 1,02 & 5 & 10,04 & 5 & 0,60 \\
\hline 10 & 1000 & 333 & 3 & 5 & 170,27 & 5 & 168,75 & 5 & 0,29 & 5 & 0,57 & 5 & 50,58 & 5 & 0,68 \\
\hline 10 & 1000 & 333 & 4 & 5 & 380,23 & 5 & 308,91 & 5 & 0,79 & 5 & 0,98 & 5 & 16,31 & 5 & 0,66 \\
\hline 10 & 1000 & 500 & 3 & 5 & 365,20 & 5 & 412,48 & 5 & 0,56 & 5 & 0,64 & 5 & 40,66 & 5 & 0,80 \\
\hline 10 & 1000 & 500 & 4 & 4 & 838,00 & 5 & 608,46 & 5 & 0,74 & 5 & 1,17 & 5 & 26,14 & 5 & 1,16 \\
\hline 10 & 2000 & 500 & 3 & 3 & 1538,42 & 3 & 1310,11 & 5 & 0,81 & 5 & 1,90 & 5 & 104,57 & 5 & 1,31 \\
\hline 10 & 2000 & 500 & 4 & 3 & 1197,88 & 4 & 1020,28 & 5 & 1,04 & 5 & 1,44 & 5 & 64,34 & 5 & 1,62 \\
\hline 10 & 2000 & 667 & 3 & 2 & 1406,81 & 2 & 1365,28 & 5 & 1,23 & 5 & 1,20 & 5 & 123,13 & 5 & 1,22 \\
\hline 10 & 2000 & 667 & 4 & 3 & 1092,84 & 4 & 928,63 & 5 & 1,01 & 5 & 1,24 & 5 & 58,13 & 5 & 1,16 \\
\hline 10 & 2000 & 1000 & 3 & 4 & 1155,24 & 4 & 1125,38 & 5 & 0,72 & 5 & 0,83 & 5 & 183,55 & 5 & 1,59 \\
\hline 10 & 2000 & 1000 & 4 & 3 & 980,54 & 3 & 1215,99 & 5 & 1,11 & 5 & 1,84 & 5 & 91,77 & 5 & 1,66 \\
\hline 10 & 5000 & 1250 & 3 & 1 & 1673,56 & 1 & 1796,25 & 5 & 2,66 & 5 & 3,00 & 3 & 1265,41 & 5 & 4,80 \\
\hline 10 & 5000 & 1250 & 4 & 0 & - & 0 & - & 5 & 9,46 & 5 & 14,29 & 5 & 421,92 & 5 & 5,76 \\
\hline 10 & 5000 & 1667 & 3 & 0 & - & 0 & - & 5 & 5,08 & 5 & 10,21 & 4 & 840,57 & 5 & 3,86 \\
\hline 10 & 5000 & 1667 & 4 & 2 & 1430,78 & 2 & 1690,69 & 5 & 2,77 & 5 & 3,19 & 4 & 627,48 & 5 & 5,12 \\
\hline 10 & 5000 & 2500 & 3 & 1 & 1568,60 & 0 & - & 5 & 3,91 & 5 & 5,34 & 4 & 754,36 & 5 & 5,44 \\
\hline 10 & 5000 & 2500 & 4 & 1 & 1727,35 & 1 & 1649,57 & 5 & 6,04 & 5 & 8,31 & 3 & 1125,20 & 5 & 5,97 \\
\hline 10 & 10000 & 2500 & 3 & 0 & - & 0 & - & 5 & 9,37 & 5 & 17,15 & 2 & 1211,56 & 5 & 10,24 \\
\hline 10 & 10000 & 2500 & 4 & 0 & - & 0 & - & 5 & 16,19 & 5 & 26,46 & 4 & 955,69 & 5 & 8,48 \\
\hline 10 & 10000 & 3333 & 3 & 0 & - & 0 & - & 5 & 17,88 & 5 & 20,49 & 1 & 1649,16 & 5 & 13,44 \\
\hline 10 & 10000 & 3333 & 4 & 0 & - & 0 & - & 5 & 19,62 & 5 & 18,73 & 3 & 1152,58 & 5 & 10,75 \\
\hline 10 & 10000 & 5000 & 3 & 1 & 1800,00 & 0 & - & 5 & 11,24 & 5 & 23,00 & 2 & 1800,00 & 5 & 10,91 \\
\hline 10 & 10000 & 5000 & 4 & 0 & - & 0 & - & 5 & 28,00 & 5 & 16,26 & 2 & 1314,08 & 5 & 14,30 \\
\hline 20 & 1000 & 250 & 3 & 4 & 797,87 & 5 & 477,74 & 5 & 0,70 & 5 & 1,38 & 5 & 41,66 & 5 & 1,25 \\
\hline 20 & 1000 & 250 & 4 & 5 & 852,01 & 5 & 548,28 & 5 & 0,64 & 5 & 1,31 & 5 & 20,22 & 5 & 0,88 \\
\hline 20 & 1000 & 333 & 3 & 4 & 608,99 & 5 & 296,04 & 5 & 0,54 & 5 & 1,03 & 5 & 29,01 & 5 & 0,99 \\
\hline 20 & 1000 & 333 & 4 & 4 & 1026,53 & 4 & 773,41 & 5 & 1,01 & 5 & 1,93 & 5 & 39,01 & 5 & 1,55 \\
\hline 20 & 1000 & 500 & 3 & 4 & 881,29 & 5 & 412,55 & 5 & 0,51 & 5 & 1,13 & 5 & 55,68 & 5 & 1,42 \\
\hline 20 & 1000 & 500 & 4 & 2 & 1499,86 & 3 & 1478,83 & 5 & 0,93 & 5 & 1,55 & 5 & 36,02 & 5 & 1,48 \\
\hline 20 & 2000 & 500 & 3 & 1 & 1629,43 & 2 & 1800,00 & 5 & 1,93 & 5 & 2,21 & 5 & 212,07 & 5 & 2,08 \\
\hline 20 & 2000 & 500 & 4 & 1 & 1623,38 & 2 & 1634,03 & 5 & 3,91 & 5 & 2,82 & 5 & 217,86 & 5 & 2,42 \\
\hline 20 & 2000 & 667 & 3 & 2 & 1612,20 & 3 & 1801,02 & 5 & 1,78 & 5 & 2,05 & 5 & 223,80 & 5 & 2,40 \\
\hline 20 & 2000 & 667 & 4 & 3 & 1421,62 & 2 & 1416,62 & 5 & 1,34 & 5 & 2,44 & 5 & 134,67 & 5 & 2,64 \\
\hline 20 & 2000 & 1000 & 3 & 0 & - & 2 & 1800,00 & 5 & 2,45 & 5 & 1,86 & 5 & 181,95 & 5 & 3,02 \\
\hline 20 & 2000 & 1000 & 4 & 1 & 1800,00 & 1 & 1800,00 & 5 & 5,09 & 5 & 3,08 & 5 & 345,70 & 5 & 3,44 \\
\hline 20 & 5000 & 1250 & 3 & 0 & - & 0 & - & 5 & 7,20 & 5 & 9,37 & 3 & 1188,15 & 5 & 8,67 \\
\hline 20 & 5000 & 1250 & 4 & 0 & - & 0 & - & 5 & 14,87 & 5 & 17,02 & 5 & 868,00 & 5 & 9,05 \\
\hline 20 & 5000 & 1667 & 3 & 0 & - & 0 & - & 5 & 7,92 & 5 & 30,75 & 2 & 1513,57 & 5 & 8,88 \\
\hline 20 & 5000 & 1667 & 4 & 0 & - & 0 & - & 5 & 7,28 & 5 & 18,85 & 5 & 1034,49 & 5 & 9,46 \\
\hline 20 & 5000 & 2500 & 3 & 0 & - & 0 & - & 5 & 16,23 & 5 & 13,53 & 2 & 2447,37 & 5 & 15,67 \\
\hline 20 & 5000 & 2500 & 4 & 0 & - & 0 & - & 5 & 20,15 & 5 & 10,45 & 2 & 1585,53 & 5 & 13,13 \\
\hline 20 & 10000 & 2500 & 3 & 0 & - & 0 & - & 5 & 52,42 & 5 & 65,91 & 2 & 1745,86 & 5 & 23,08 \\
\hline 20 & 10000 & 2500 & 4 & 0 & - & 0 & - & 5 & 96,02 & 5 & 63,30 & 1 & 1800,00 & 5 & 27,02 \\
\hline 20 & 10000 & 3333 & 3 & 0 & - & 0 & - & 5 & 28,93 & 5 & 25,17 & 2 & 1750,37 & 5 & 22,32 \\
\hline 20 & 10000 & 3333 & 4 & 0 & - & 0 & - & 5 & 125,00 & 5 & 93,66 & 0 & - & 5 & 29,79 \\
\hline 20 & 10000 & 5000 & 3 & 0 & - & 0 & - & 5 & 40,39 & 5 & 85,61 & 2 & 1800,00 & 5 & 38,66 \\
\hline 20 & 10000 & 5000 & 4 & 0 & - & 0 & - & 5 & 88,29 & 5 & 167,54 & 1 & 1800 & 5 & 27,76 \\
\hline
\end{tabular}

TABLE 2. Numerical results for BLP under the $\ell_{1}$ and $l_{\infty}$ norm. 


\section{REFERENCES}

[1] Albareda-Sambola, M., Fernández, E., Hinojosa, Y., and Puerto, J. (2009). The multi-period incremental service facility location problem. Computers \& Operations Research, 36(5), 13561375 .

[2] Albareda-Sambola, M., Hinojosa, Y., and Puerto, J. (2015). The reliable p-median problem with at-facility service. European Journal of Operational Research, 245(3), 656-666.

[3] Blanco, V., Puerto, J., Ali, SEHB. (2014). Revisiting several problems and algorithms in continuous location with $l_{\tau}$ norms. Computational Optimization and Applications. 58(3), 563595 .

[4] Bélanger, V., Ruiz, A., and Soriano, P. (2019). Recent optimization models and trends in location, relocation, and dispatching of emergency medical vehicles. European Journal of Operational Research. 272 (1), 1-23.

[5] Berman, O., Drezner, Z., and Krass, D. (2010). Generalized coverage: New developments in covering location models. Computers \& Operations Research, 37(10), 1675-1687.

[6] Boonmee, C., Arimura, M., and Asada, T. (2017). Facility location optimization model for emergency humanitarian logistics. International Journal of Disaster Risk Reduction, 24, 485498.

[7] Brimberg, J., and Juel, H. (1998) A bi-criteria model for locating a semi-desirable facility in the plane. European Journal of Operational Research 106 (1), 144-151.

[8] Church, R.L., and Scaparra M.P. (2007). Protecting critical assets: the r-interdiction median problem with fortification Geographical Analysis 39 (2), 129-146

[9] Aksen, D., and Aras, N. (2012). A bilevel fixed charge location model for facilities under imminent attack. Computers \& Operations Research, 39(7), 1364-1381.

[10] Erkut, E., and Neuman, S. (1989) Analytical models for locating undesirable facilities. European Journal of Operational Research 40 (3), 275-291.

[11] Garey, M.R., and Johnson D.S. (1979).Computers and intractability. A guide to the theory of NP-Completeness. W.H. Freeman.

[12] Hammad, A. W., Rey, D., and Akbarnezhad, A. (2018). A Bi-level Mixed Integer Programming Model to Solve the Multi-Servicing Facility Location Problem, Minimising Negative Impacts Due to an Existing Semi-Obnoxious Facility. Data and Decision Sciences in Action, 381-395. Springer.

[13] Kalcsics, J., Nickel, S., Pozo, M. A., Puerto, J., and Rodríguez-Chía, A. M. (2014). The multicriteria p-facility median location problem on networks. European Journal of Operational Research, 235(3), 484-493.

[14] Labbé, M., Peeters, D., and Thisse, J. F. (1995). Location on networks. Handbooks in operations research and management science, 8, 551-624. 
[15] Love, R. F., Morris, J. G. and Wesolowsky, G. O. (1998). Facilities location: models and methods. North-Holland, New York.

[16] Luenberger, D. G., and Ye, Y. (1984). Linear and nonlinear programming (Vol. 2). Section 6.2. Reading, MA: Addison-wesley.

[17] McCormick, G. P. (1976). Computability of global solutions to factorable nonconvex programs: Part I Convex underestimating problems. Mathematical programming 10, 147?175.

[18] Melachrinoudis,E., and Xanthopulos, Z. (2003) Semi-obnoxious single facility location in Euclidean space. Computers \& Operations Research 30, 2191-2209.

[19] Nickel, S., and Puerto, J. (2006). Location theory: a unified approach. Springer Science and Business Media.

[20] Nickel, S. and Puerto, J. (2014). A unified approach to location analysis: Ordered Median Problems. Springer.

[21] Owen, S. H., and Daskin, M. S. (1998). Strategic facility location: A review. European journal of operational research, 111(3), 423-447.

[22] Rockafellar, R. T. (1970). Convex analysis. Princeton university press.

[23] Scaparra M.P., and Church, R.L. (2008). A bilevel mixed-integer program for critical infrastructure protection planning. Computers \& Operations Research 35 (6), 1905-1923

[24] Schmid, V., and Doerner, K. F. (2010). Ambulance location and relocation problems with time-dependent travel times. European journal of operational research, 207(3), 1293-1303. 